\title{
Power Scaling Concept for Solid-State Lasers Based on a Rotating Cavity Configuration
}

\author{
M. Eckold, J. I. Mackenzie, W. A. Clarkson \\ Optoelectronics Research Centre, University of Southampton, Southampton, SO17 1BJ, UK \\ matt.eckold@soton.ac.uk
}

\begin{abstract}
A laser architecture for scaling output power and avoiding deleterious thermal effects based on a resonator with a rotating periscope is described. Preliminary results for continuouswave and Q-switched operation are presented.

OCIS codes: (140.3480) Lasers, diode-pumped; (140.3580) Lasers, solid-state
\end{abstract}

\section{Introduction}

The power scaling potential of solid state lasers is limited by their ability to handle the thermal load generated in the laser medium during the pumping cycle. The temperature gradients that arise from the waste heat lead to a degradation in beam quality due to thermal lensing, reduced efficiency and, eventually, catastrophic failure. A number of different schemes to overcome these limitations have been developed; some are based on managing the direction of heat flow from the pumped volume to the coolant, to prevent transverse temperature gradients; such as in a thin disk laser [1]. Others lower the pump density whilst increasing the pumped volume, yielding lower temperature rises and a larger heat sinking potential; this technique is best demonstrated by the fibre laser.

Alternatively, laser action can be separated from any thermal processes by making use of the different time scales associated with lasing and heat flow. In a quasi-CW laser the laser energy can be extracted before thermal diffusion can produce a temperature gradient [2]. Instead of separating these two processes in time it is possible to separate them in space. Moving slab lasers and the Rotary Disk Laser, RDL, do this by moving the gain medium relative to the laser mode $[3,4]$. However, a moving gain medium requires complicated heat sinking arrangements, in the case of the RDL gaseous helium is used as a coolant [4].

Here, we present a different approach for overcoming the thermal limitations based on a concept we refer to as the Rotating Cavity Laser (RCL). Like the RDL, the RCL involves the relative motion of the laser mode and heated gain medium to prevent detrimental thermal effects. However, in an RCL the laser medium is stationary and the motion is instead provided by rotating an intra-cavity periscope. This periscope steers a collinear pump beam and laser mode over the gain medium in a circular trajectory, see Fig. 1, allowing a simple, face cooled, heat sinking arrangement to be used. Not only does this simplify the mechanical design it also relaxes the tolerances compared to the RDL, but, by virtue of allowing effective face cooling, transverse heat flow is further reduced.

By removing the thermal load in this manner high pump densities can be used and thus smaller pumped regions than those found in fibre and thin disks lasers. Energy stored in the gain medium is therefore less susceptible to extraction via ASE, allowing the generation of high energy pulses at high repetition rates. Furthermore, the short interaction length and flexibility in mode size in an RCL makes the handling of high peak powers straight forward, unlike in a fibre laser.

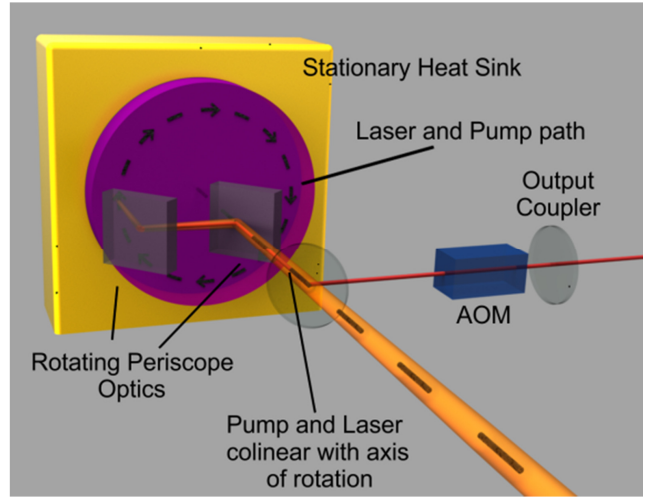

Fig. 1. Schematic of Q-switched RCL

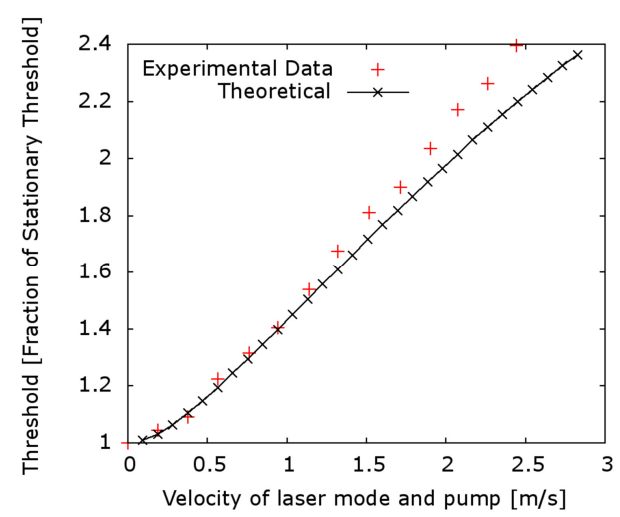

Fig. 2. Effect of motion on laser performance at threshold 


\section{Effect of Motion}

An approximation for the temperature rise in a RDL has been derived by Basu in [5], and is also applicable to the RCL. It shows that the temperature rise is dependent on the velocity of the pump spot relative to the gain medium, which gives a lower bound on the required velocity. As the motion is circular, the thermal load handling ability of an RCL can be scaled by either increasing the radius of rotation or the rotation frequency. This provides a low cost power scaling mechanism.

Whilst the relative motion of the laser mode and gain medium removes heat from the lasing area, it also has the potential to move excited ions out of the lasing region. By adapting the 4-level rate equations to include motion it is possible to quantify this effect for Nd:YAG. Fig. 2 shows that the predicted change in threshold when this new loss mechanism is taken into account is in good agreement with experimentally measured values. By operating the RCL at a level well above threshold, stimulated emission becomes the dominant energy extraction mechanism; in this regime the effect of rotation can be ignored due to the much faster extraction rate. A RCL thus needs to be operated at a power level high enough to prevent reduced energy extraction due to motion, whilst moving fast enough to prevent the associated detrimental thermal effects. Equally, the repetition rate of a RCL in the pulsed regime must be fast compared to the velocity of the laser mode. This design constraint will be discussed.

\section{Results}

To demonstrate the Q-switched operation of the RCL the resonator in Fig 1 was employed. This used a $35 \mathrm{~mm}$ diameter, $6 \mathrm{~mm}$ thick ceramic Nd:YAG slab, pumped with a $808 \mathrm{~nm}$, fibre-coupled diode. The rotating periscope introduced a displacement of $15 \mathrm{~mm}$ from the rotation axis. The output coupler was $70 \%$ reflective and the calculated $\mathrm{TEM}_{00}$ waist inside the Nd:YAG slab was $500 \mu \mathrm{m}$. When operated without the Q-switch present a maximum output power of $16 \mathrm{~W}$ was observed when the periscope was rotated with a period of $200 \mathrm{~ms}$ (see Fig. 3). This was only limited by the available pump power and there was no evidence of a thermally induced roll-over. Reducing the period of rotation to $100 \mathrm{~ms}$ had no effect on the maximum output power, indicating losses due to the motion of the laser mode are negligible. When the periscope was kept stationary thermal lensing made the cavity unstable and the output power was limited to $<1 \mathrm{~W}$.

An AOM was placed in the resonator and resonator was pumped with $55 \mathrm{~W}$ of absorbed power whilst the periscope was rotating with a period of $200 \mathrm{~ms}$. A maximum pulse energy of $2.4 \mathrm{~mJ}$ was recorded at a repetition rate of $1 \mathrm{kHz}$, with a pulse width of $57 \mathrm{~ns}$ (see Fig. 4). The prospects for further improvement in performance of the RCL will be discussed along with preliminary results from a thermal FEA model.

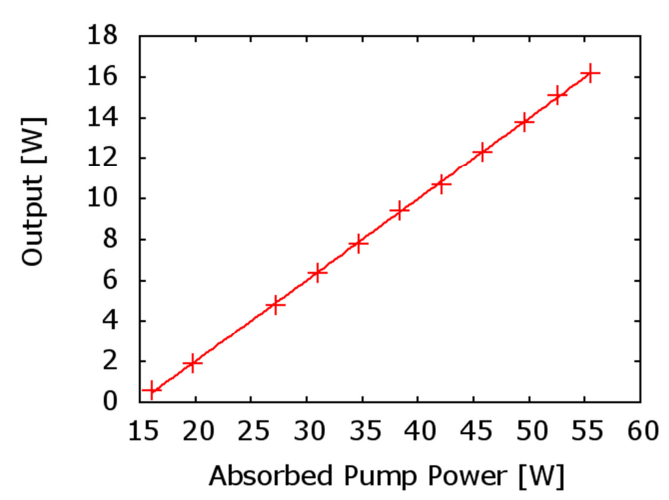

Fig. 3. CW performance

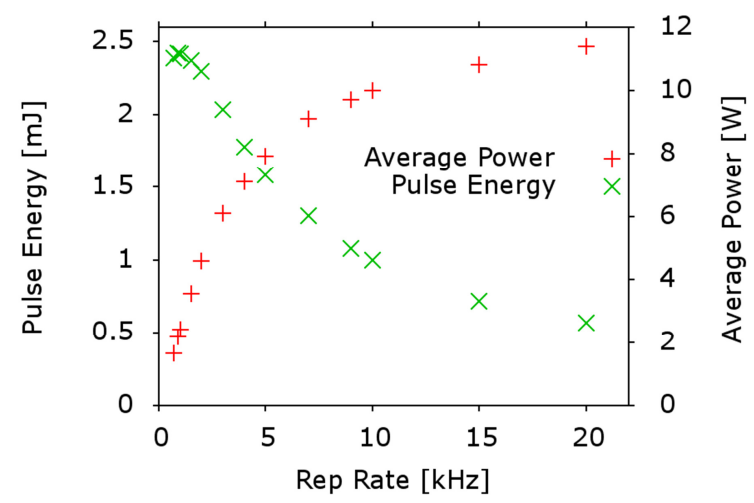

Fig. 4. Q-switched performance with 55W of absorbed power

\section{References}

[1] A. Giesen and J. Speiser, "Fifteen Years of Work on Thin-Disk Lasers: Results and Scaling Laws," IEEE Journal of Selected Topics in Quantum Electronics, vol. 13, no. 3, pp. 598-609, 2007.

[2] Koechner, W. (2006). Solid State Laser Engineering (6th ed.). New York: Springer.

[3] E. Stappaerts, "Hybrid Heat capacity-moving slab laser concept," Tech. Rep. April, Lawrence Livermore National Laboratory (LLNL), Livermore, CA, 2002.

[4] S. Basu, "Nd-YAG and Yb-YAG rotary disk lasers," IEEE Journal of Quantum Electronics, vol. 11, no. 3, pp. 626-630, 2005.

[5] S. Basu and R. L. Byer, "Diode-pumped moving-disc laser: a new configuration for high average power generation," Optical and Quantum Electronics, vol. 22, pp. S33-S37, July 1990. 